\title{
Prediction of the atomic structure of two-dimensional materials on substrates
}

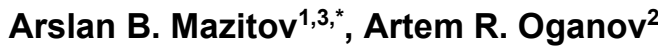

\author{
${ }^{1}$ Moscow Institute of Physics and Technology, 9 Institutsky lane, Dolgoprudny 141701, Russian Federation, ${ }^{2}$ Skolkovo Institute of \\ Science and Technology, Skolkovo Innovation Center, 3 Nobel Street, Moscow 121205, Russian Federation, ${ }^{3}$ Dukhov Research \\ Institute of Automatics (VNIIA), Moscow 127055, Russian Federation \\ *arslan.mazitov@phystech.edu
}

Today the study of two-dimensional (2D) materials has become one of the key objectives of materials science. Unlike their threedimensional counterparts, 2D materials can simultaneously demonstrate unique transport and mechanical properties due to their dimensionality and quantum size effect. Weak van der Waals interaction between layers in heterostructures of 2D materials, electron confinement inside the layers, and high surface-to-volume ratio lead to remarkable changes in electronic and optical properties of the materials, as well as in their chemical and mechanical response. Besides, a wide range of ways to tune properties using lateral and vertical heterostructures fabrication, chemical functionalization, strain, defect and substrate engineering, makes 2D materials ideal candidates for developing a new class of electronic devices.

In their fabrication and application, 2D materials are usually located on top of the substrate or combined into heterostructures, which makes their structures and properties strongly depend on the nature and quality of the environment. Here, we present a novel method for studying the atomic structures of two-dimensional materials and epitaxial thin films on arbitrary substrates. The method can predict successful stages of epitaxial growth and the regions of stability of each atomic configuration with experimental parameters of interest (Figure 1). We demonstrate the performance of our methodology in the prediction of the atomic structure of $\mathrm{MoS}_{2}$ on $\mathrm{Al}_{2} \mathrm{O}_{3}$ (0001) substrate. The method is also applied to study the CVD growth of graphene and hexagonal boron nitride on Cu (111) substrates. In both cases, stable monolayer and multilayer structures were found. The stability of all the structures in terms of partial pressures of precursors and temperature of growth is predicted within the ab initio thermodynamics approach.

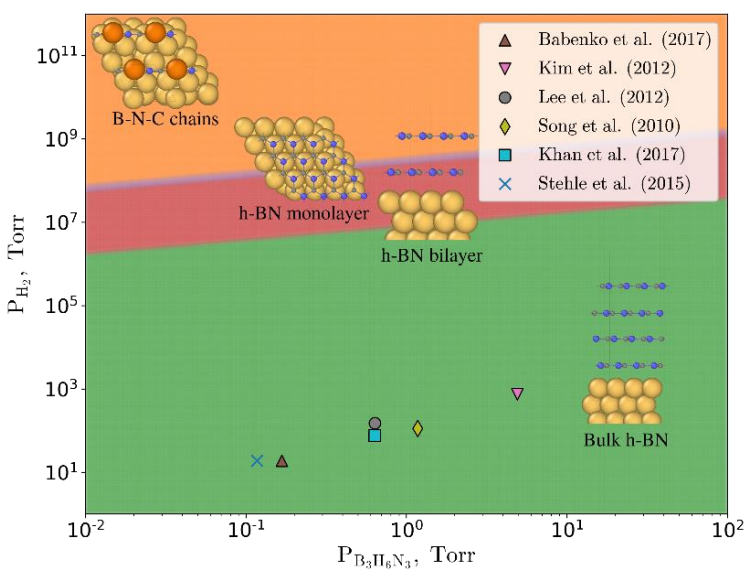

Figure 1. Predicted stable structures of $\mathrm{BN} / \mathrm{Cu}(111)$ system and phase diagram of their CVD growth at $\mathrm{T}=1000{ }^{\circ} \mathrm{C}$. $\mathrm{Regions}$ of stability of different structures are represented with colors. Four successful stages of h-BN synthesis: B-N-C chains, h-BN monolayer, bilayer and bulk representative were found in the calculation.

Keywords: 2D materials; first principles; crystal structure prediction; CVD growth; phase diagram 